\title{
Lateral force effects of three-axle locomotive bogie on track
}

\author{
T. Michálek ${ }^{a, *}$, L. Haupt ${ }^{a, b}$, J. Zelenka ${ }^{a}$, M. Kohout ${ }^{a}$, S. Liberováa ${ }^{a}$ \\ ${ }^{a}$ University of Pardubice, Faculty of Transport Engineering, Department of Transport Means and Diagnostics, Section of Rail Vehicles; \\ Detached Branch of the Faculty of Transport Engineering, Nádražní 547, 56002 Česká Třebová, Czech Republic \\ ${ }^{b}$ CZ LOKO, a.s., Semanínská 580, 56002 Česká Třebová, Czech Republic \\ Received 26 February 2018; accepted 8 June 2018
}

\begin{abstract}
This paper deals with guiding behaviour of a diesel-electric locomotive equipped with new three-axle bogies which were developed in co-operation of the company CZ LOKO and the Faculty of Transport Engineering of the University of Pardubice. At first, the design of the new bogie intended for the track gauge 1520 mm is shortly introduced. Then, the attention is paid to three different variants of secondary suspension design and their influence on lateral force effects of the locomotive on track in curves. The assessment is performed by means of the multibody simulation tool "SJKV" and it takes into account GOST standards. Possibilities of application of a system of active elements for bogie steering are also evaluated.
\end{abstract}

(C) 2018 University of West Bohemia. All rights reserved.

Keywords: three-axle bogie, secondary suspension, flexi-coil spring, tilting pad, active element, guiding force, GOST standards, safety against derailment, multi-body simulation

\section{Introduction}

In years 2015 to 2017, Faculty of Transport Engineering of the University of Pardubice participated on the R\&D project No. TH01010455 of the Technology Agency of the Czech Republic solved by the company CZ LOKO, a.s. The aim of this project was development of a new three-axle bogie intended for a six-axle diesel-electric locomotive for the track gauge $1520 \mathrm{~mm}$ including manufacturing of a bogie prototype (see [5]).

Generally, a well-known problem of standard three-axle locomotive bogies is a high level of lateral force interaction of the vehicle on the track in curves as well as a lower level of the safety against derailment (in comparison with common two-axle bogies). Therefore, some special technical solutions leading to an improvement of dynamic properties of the three-axle bogies (e.g. lateral free play of the intermediate wheelset, mechanical systems for radial bogie steering, using of special suspension elements in secondary suspension stage etc.) are applied in modern bogie design (see, e.g., $[1,11]$ ).

During the development of the new bogie, a special attention was paid to the design solution of secondary suspension and connection between the vehicle body and the bogie. In the secondary suspension stage, two sets of flexi-coil springs in combination with tilting rubber-metal pads are proposed for use on each bogie. The tilting pad allows reaching of acceptable values of stress in the spring material at the horizontal deformation of the spring/pad assembly, i.e. especially at the rotation of the bogie relatively to the vehicle body if the flexi-coil effect is used. The second effect of application of the tilting pads under the secondary springs is their significant and directionally dependent influence on the horizontal stiffness.

\footnotetext{
${ }^{*}$ Corresponding author. Tel.: +420 466037 428, e-mail: tomas.michalek@upce.cz. https://doi.org/10.24132/acm.2018.436
} 
The horizontal stiffness characteristics of the spring/pad assembly are strongly dependent on type of the used pad. Therefore, an influence of two different types of the tilting pads on the dynamic properties of the whole vehicle is observed in this work. Although some analytical methods of estimation of the horizontal stiffness characteristics of flexi-coil springs with pads exist (see, e.g., $[2,7,8]$ ), their application is problematic because of required detailed knowledge of parameters of the pads. Besides to that, the analytical calculations usually provide only approximate results and different calculation methods provide different results. Therefore, the horizontal stiffness characteristics of the investigated spring/pad assemblies were determined experimentally on the dynamic test stand of the Faculty of Transport Engineering in Pardubice and subsequently used as input data for the multi-body simulations.

\section{Description of design solution of the new bogie}

The new three-axle bogie CZ LOKO for the track gauge $1520 \mathrm{~mm}$ consists of a welded frame and three wheelsets with a wheelbase of $2 \times 1850 \mathrm{~mm}$. The wheelsets are guided in the bogie frame by means of longitudinal rods; the primary suspension is realized by a couple of flexi-coil springs per axle box and supplemented with a vertical hydraulic damper situated in one of the primary springs. Lateral and vertical bump stops are solved in the top part of individual axle boxes. The wheels with a nominal diameter of $1100 \mathrm{~mm}$ are equipped with disc mounted brake discs. Traction drive is ensured by individually controlled axle-mounted asynchronous traction motors with a maximum power of $3 \times 366 \mathrm{~kW}$.

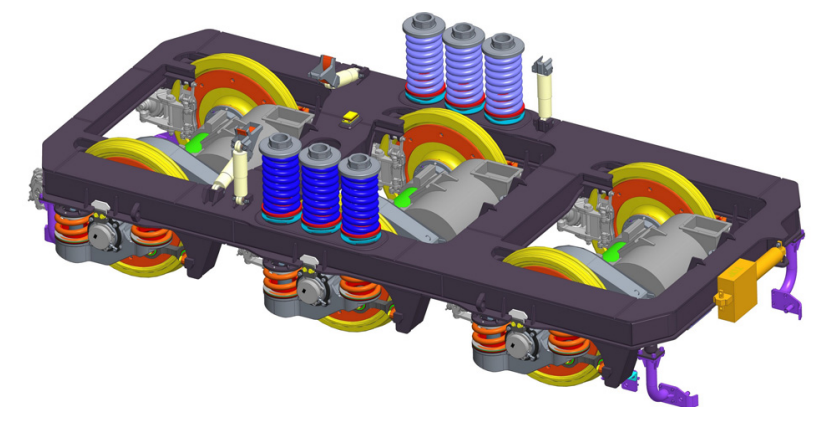

Fig. 1. Visualisation of the new three-axle bogie $\mathrm{CZ}$ LOKO

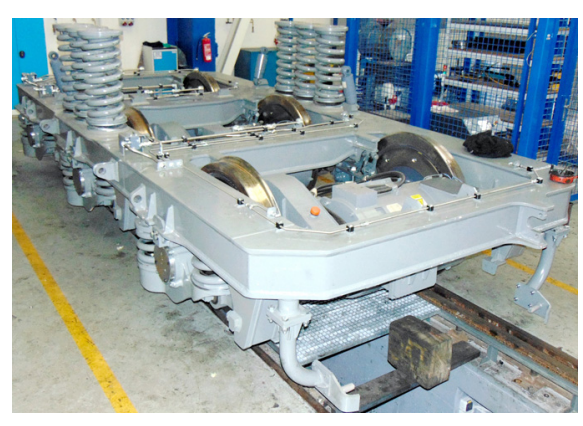

Fig. 2. Prototype of the new bogie

The longitudinal force transmission between the bogie frame and the vehicle body is performed by means of an eccentrically situated (longitudinally shifted) pivot. The secondary suspension consists of three flexi-coil springs situated on each longitudinal beam of the bogie frame. On the bottom end of the springs, the tilting rubber-metal pads are proposed for use. These pads are oriented in such way that their axes of tilting head to the centre of the pivot for longitudinal force transmission. This solution ensures minimization of the bogie yaw resistance. The secondary suspension is supplemented by lateral and vertical hydraulic dampers as well as lateral and vertical bump stops. Optionally, the bogie can be equipped with a laterally situated active element for bogie steering in small-radius curves on the front beam of the bogie frame; this active element can also serve as a bogie yaw damper during run of the vehicle in straight track. The proposed design solution of the connection between the vehicle body and the bogie frame is registered as a protected technical solution (see [6]). A 3D model of the bogie is presented in Fig. 1; in Fig. 2, a finished bogie prototype in shown. The total mass of the new three-axle bogie is approximately $19 \mathrm{t}$; its more detailed description can be found in [5]. 


\section{Possible arrangements of secondary suspension}

During the development of the bogie, three versions of arrangement of the secondary suspension were considered for a virtual locomotive with total mass of $138 \mathrm{t}$ (i.e. axle load of $23 \mathrm{t}$ ) and $150 \mathrm{t}$ (i.e. axle load of $25 \mathrm{t}$ ). Each of them shows different properties from the point of view of guiding behaviour of the locomotive and possible application of active elements for bogie steering.

The first variant is the application of flexi-coil springs without the tilting pads. This solution shows a high value of the bogie yaw resistance and the spring material is intensively loaded at the movement of the bogie relative to the vehicle body, especially at the bogie rotation. The high value of the bogie yaw resistance leads to a high quasistatic guiding force acting on the outer wheel of the first wheelset in small-radius curves; the stress in spring material limits maximum allowable deformation of the springs in horizontal plane and therefore also the minimum curve radius in which the locomotive can be operated. This limitation depends also on dimensional parameters of the vehicle, i.e. on the distance between bogie pivots.

The second variant is the application of tilting rubber-metal pads with cylindrical tilting surface (pad "A") under the flexi-coil springs. This variant (see Fig. 3) shows a very significant difference between the stiffness of the spring/pad assembly in the direction, in which the pad allows tilting ( $0 \mathrm{deg})$, and in the perpendicular direction $(90 \mathrm{deg})$. Whereas the lateral stiffness in the direction " $90 \mathrm{deg}$ " is close to the lateral stiffness of the spring itself, the lateral stiffness in the direction " 0 deg" can be zero or even negative for higher values of vertical load of the spring/pad assembly. Therefore, this solution is characterized with a maximum reduction of the bogie yaw resistance at rotation of the bogie relatively to the vehicle body in laterally centred position. Properties of springs with this type of pads (including their influence on guiding behaviour of a standard four-axle locomotive in small-radius curves) are also an object of paper [9].
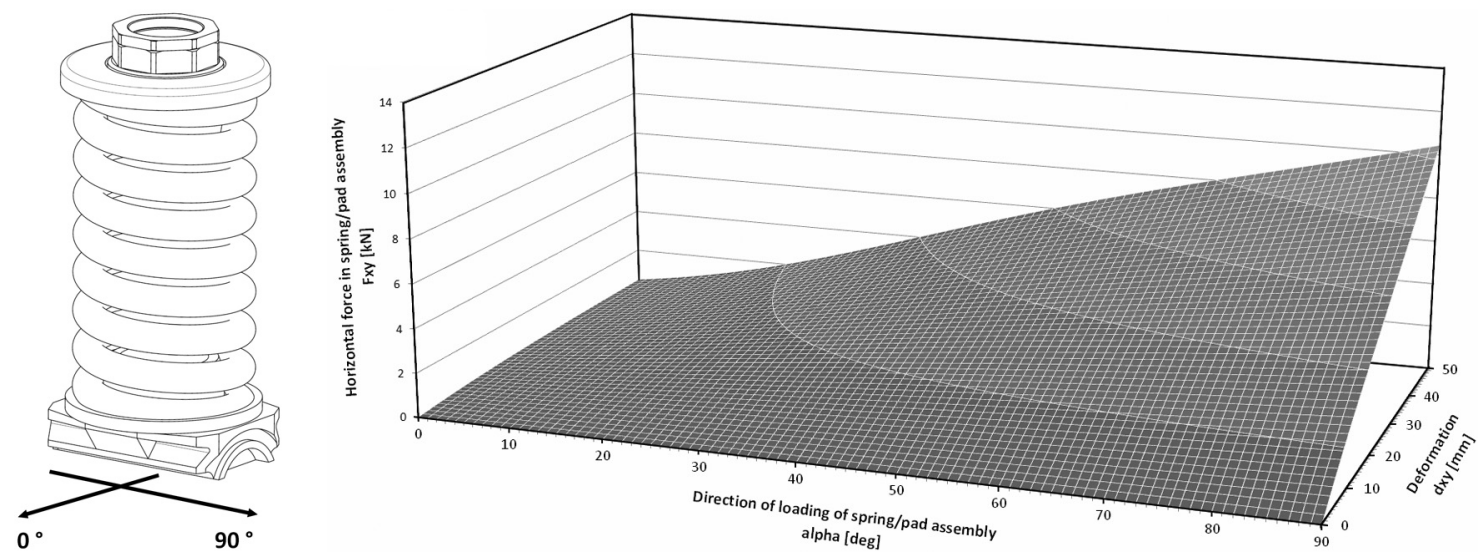

Fig. 3. Secondary flexi-coil spring with tilting rubber-metal pad "A" (left) and horizontal characteristic of the spring/pad assembly for the vertical load of $82.5 \mathrm{kN}$ (right)

The last observed alternative is the application of tilting rubber-metal pads with specially shaped tilting surface (pad "B") under the flexi-coil springs. In comparison with the pad "A", this variant (see Fig. 4) is characterized with a more significant decrease of the lateral stiffness of the spring/pad assembly in the direction "90 deg" but not so significant decrease of its lateral stiffness in the direction " 0 deg".

The horizontal characteristics of the considered spring/pad assemblies depicted in Figs. 3 and 4 were obtained as a mathematical approximation of the measured horizontal force $F_{x y}$ 

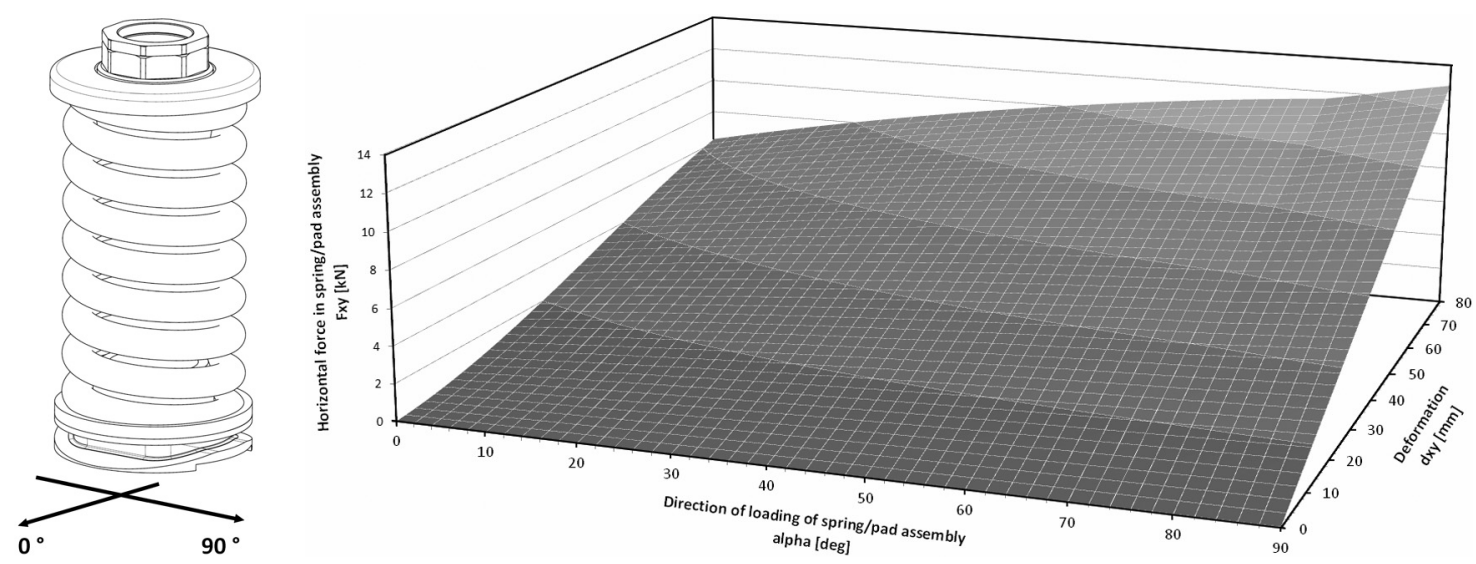

Fig. 4. Secondary flexi-coil spring with tilting rubber-metal pad "B" (left) and horizontal characteristic of the spring/pad assembly for the vertical load of $82.5 \mathrm{kN}$ (right)

in dependence on the vertical load $F_{z}$ of the spring/pad assembly, the direction $\alpha$ of loading relatively to the pad orientation and the horizontal deformation $\Delta_{x y}$ of the spring with pad as follows

$$
F_{x y}=f\left(F_{z}, \alpha, \Delta_{x y}\right)
$$

In Figs. 3 and 4, only the characteristics for the considered static vertical load of the spring/pad assembly of $82.5 \mathrm{kN}$ are presented. As a particular example of the approximation, a function defining the horizontal characteristic of the investigated flexi-coil spring in combination with the tilting pad "B" can be introduced - the horizontal force $F_{x y}$ is expressed as a polynomial function of the horizontal deformation $\Delta_{x y}$ in the form

$$
F_{x y}=C_{3} \cdot \Delta_{x y}^{3}+C_{2} \cdot \Delta_{x y}^{2}+C_{1} \cdot \Delta_{x y}
$$

where the coefficients $C_{i}$ are defined in dependence on the direction of loading $\alpha$ as

$$
C_{i}=B_{i 2} \cdot \alpha^{2}+B_{i 1} \cdot \alpha+B_{i 0}
$$

and the dependence on the vertical load $F_{z}$ of the spring/pad assembly is defined by means of the coefficients $B_{i j}$

$$
B_{i j}=A_{i j 2} \cdot F_{z}^{2}+A_{i j 1} \cdot F_{z}+A_{i j 0}
$$

Therefore, the system of coefficients $A_{i j k}$ defines the general approximation function (1) and the calculation of these constants for the particular case is based on application of least square method on the test results. A comparison of the experimentally determined horizontal characteristics of the relevant flexi-coil spring with tilting pad "B" with the above described mathematical approximation is shown in graphs in Fig. 5. In the left graph, a force-deformation dependence is presented for various directions of loading under the condition of vertical load of $85 \mathrm{kN}$; in the right graph, a dependence of the force on the direction of loading is depicted for various vertical loads under the condition of deformation of $50 \mathrm{~mm}$. The observed differences between the measured and approximated values of horizontal force in the spring/pad assembly for given conditions range in order of tenths of $\mathrm{kN}$, i.e. approximately up to $10 \%$. 

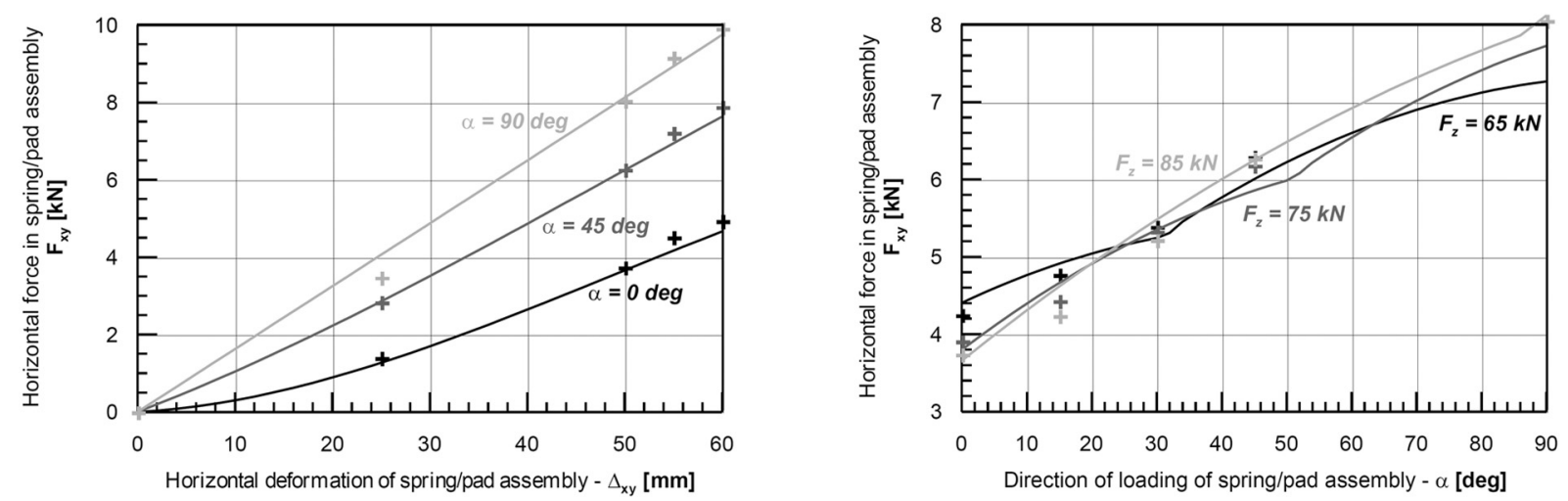

Fig. 5. Comparison of measured horizontal characteristics (points) of flexi-coil spring with tilting rubbermetal pad "B" with its mathematical approximation (lines) used in multi-body simulations

\section{Simulation results and their evaluation}

For purposes of this work, relevant simulations were performed with models of different versions of the locomotive with a total weight of $138 \mathrm{t}$ (i.e. $23 \mathrm{t}$ per axle) running through a superelevated right-hand curve with radius of $350 \mathrm{~m}$. From the point of view of basic dimensional parameters of the vehicle, the distance between bogie pivots of $9.7 \mathrm{~m}$ is considered. The speed of $89 \mathrm{~km} / \mathrm{h}$ corresponds to the unbalanced lateral acceleration of $0.8 \mathrm{~m} / \mathrm{s}^{2}$ defined as a test condition in relevant GOST standards. The wheel/rail contact conditions are defined with the nominal conical wheel profile according to GOST 11018-2011 [3] and rail profiles R65 with inclination $1: 20$ (see the roll radius difference function and the contact angle difference function of this contact pair in Fig. 6); the friction coefficient in wheel/rail contact of 0.4 represents dry rails.
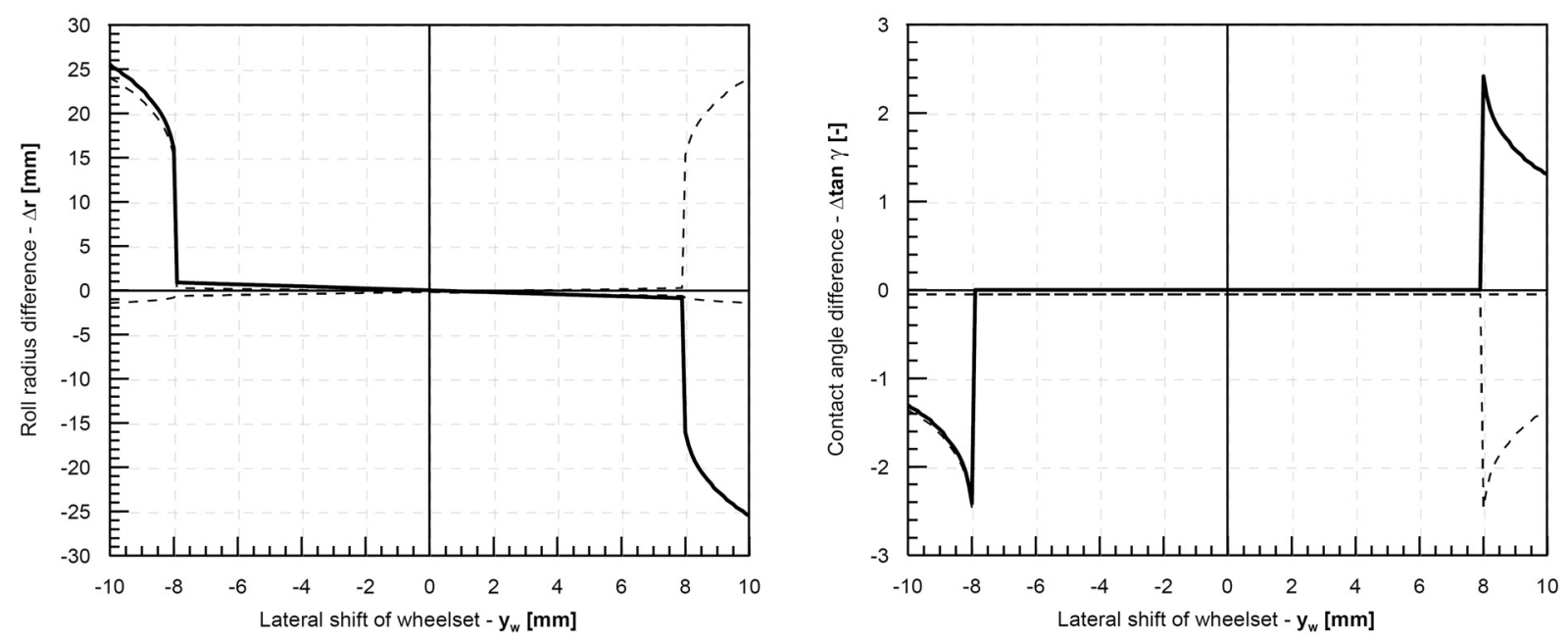

Fig. 6. Characteristics of wheel/rail contact geometry of wheelset with conical wheel profiles according to GOST 11018-2011 [3] and track with theoretical rail profiles R65 with inclination $1: 20$ and nominal track gauge

The simulations were performed by means of a new version of the original multi-body simulation tool "SJKV-CZL3nP v.3" developed at the Faculty of Transport Engineering of the University of Pardubice. In the current version, the vehicle model is represented as a mechanical system with totally 126 degrees of freedom assigned to 27 rigid bodies (wheelsets, axle boxes, 
nose-suspended traction motors, bogie frames and vehicle body). Generally, the architecture of the system "SJKV" is based on program units solving individual problems of the multi-body simulation (deformation of joints, joint forces, wheel/rail contact forces including geometric wheelset/track relationship and adhesion, accelerations of individual rigid bodies, numerical integration of equations of motion etc.). The wheel/rail contact is modelled as one-point and rigid and defined by means of characteristics of wheel/rail contact geometry. A more detailed description of the simulation software "SJKV" can be found in papers $[10,12]$.

In comparison with "standard" multi-body vehicle models, the most important particularities of the force joints applied in the system "SJKV-CZL3nP" consist in the used models of secondary suspension (consisting of the flexi-coil springs with rubber-metal tilting pads) as well as a model of active elements for radial bogie steering. The above mentioned mathematical descriptions of non-linear stiffness characteristics of the investigated spring/pad assemblies are implemented in a form of relevant functions in the program unit solving the force joints between individual bodies (bogie frames and the vehicle body). The proposed system of active elements for bogie steering in small-radius curves uses two electro-hydraulic active elements situated laterally on the outer front beams of individual bogie frames (see the scheme in Fig. 7). These active elements can exert a tensile or compressive force of a constant magnitude and therefore, they can help to steer the bogies into a position with a smaller angle of attack. The function (activation/deactivation) of the active elements is controlled on basis of signals from integrated position sensors giving information about rotation of the bogies. If a limit value of deformation for activation/deactivation of the active element is reached, the relevant active element starts/stops exerting the force of a predefined magnitude. A more detailed description of the proposed system of active elements is presented in [5].

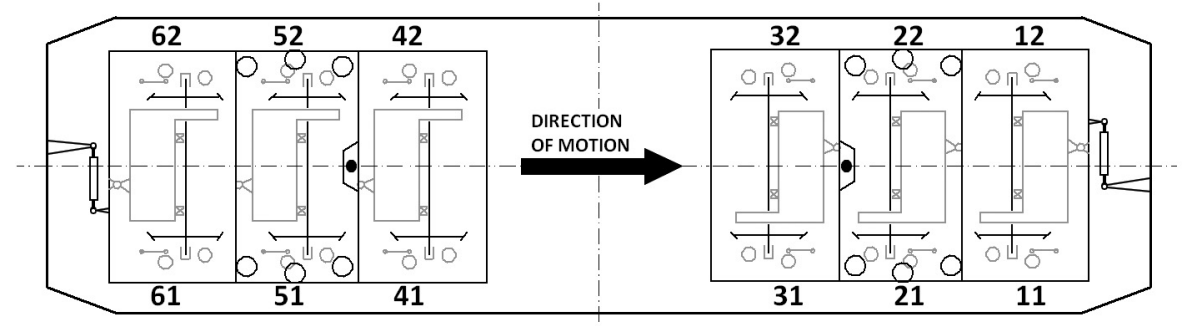

Fig. 7. Scheme of investigated locomotive with active elements for bogie steering, including numbering of individual wheels

\subsection{Influence of tilting pads under secondary springs on guiding behaviour of locomotive}

In the first stage, an influence of both types of the tilting rubber-metal pads under the secondary flexi-coil springs on guiding behaviour of the investigated locomotive was observed. In Fig. 7, there is depicted a scheme of the locomotive including the used numbering of individual wheels. The simulation results in a form of reached values of quasistatic guiding force acting on individual wheels during the run of the vehicle through the considered curve are presented in Fig. 8. For purposes of assessment of the influence of the tilting pads " $A$ " and "B" (without the contribution of the system of active elements for bogie steering), only the right (black) bar from each triplet of bars is relevant for individual wheels.

The application of the tilting pads leads to decrease of the quasistatic guiding force acting on the leading wheel of the front bogie (wheel 12) and increase of the force on the leading wheel of the rear bogie (wheel 42) in comparison with the version without pads. The quasistatic 


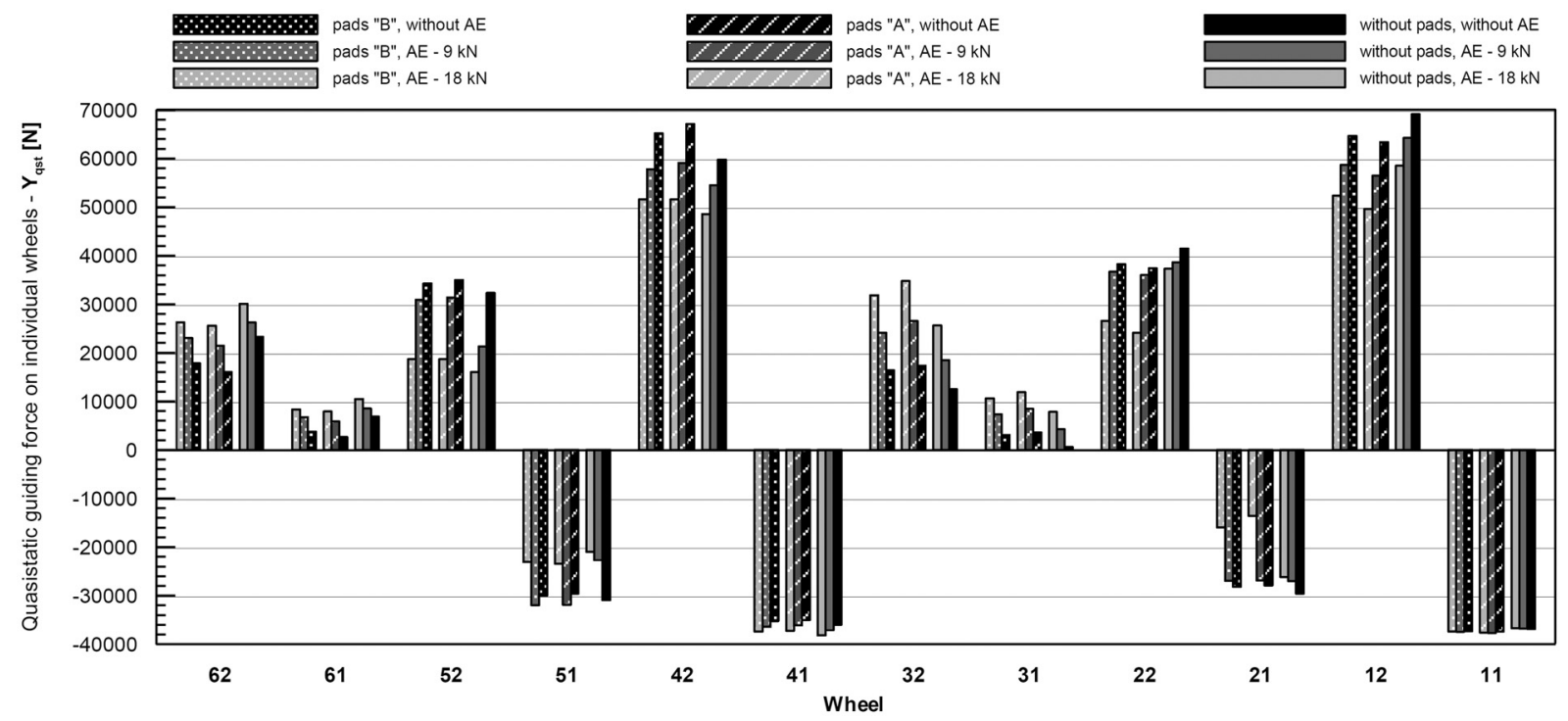

Fig. 8. Quasistatic guiding forces on individual wheels of investigated locomotive with the total weight of $138 \mathrm{t}$ in various versions (type of tilting pads, force of active elements) during the run of the locomotive through a right-hand curve with radius of $350 \mathrm{~m}$ at the unbalanced lateral acceleration of $0.8 \mathrm{~m} / \mathrm{s}^{2}$

guiding forces on the wheel 12 reach following values: $69.1 \mathrm{kN}$ (without tilting pads), $63.3 \mathrm{kN}$ (pads "A") and $64.6 \mathrm{kN}$ (pads "B"); on the wheel 42: $59.8 \mathrm{kN}$ (without tilting pads), $67.1 \mathrm{kN}$ (pads " $\mathrm{A}$ ") and $65.2 \mathrm{kN}$ (pads "B"). These results correspond to the reduction of bogie yaw resistance (as a consequence of the pad application) in combination with the proposed bogie concept with longitudinally shifted bogie pivots (the moment of leading force on wheel 12 to relevant bogie pivot is higher than the corresponding moment of leading force on wheel 42).

The changes of reached values of quasistatic guiding force are also observable on the other (not-leading) wheels but these are not so significant because of markedly lower absolute value of the force. In order to assess a level of wear in wheel/rail contact, friction power $P_{f}$ was used. This quantity can be defined for each wheel/rail contact point as

$$
P_{f}=\left(\left|T_{x} \cdot \vartheta_{x}\right|+\left|\frac{T_{y} \cdot \vartheta_{y}}{\cos \gamma}\right|\right) \cdot v
$$

where $T_{x}$ and $T_{y}$ are longitudinal and lateral creep forces in wheel/rail contact, $\vartheta_{x}$ and $\vartheta_{y}$ are relevant relative longitudinal and lateral creepages, $\gamma$ is an actual inclination of contact plane and $v$ is the vehicle speed. In case of assessment a sum of friction power values on all wheels of the vehicle, the version of the locomotive without the tilting pads shows under the considered conditions the total friction power of $37.3 \mathrm{~kW}$ and the application of both types of pads leads to a reduction of this value by approximately $1.5 \%$.

Because the tilting pads influence not only the bogie yaw resistance but also the stiffness of lateral secondary suspension, lateral deformations of the connection between the vehicle body and individual bogie frames as well as the roll coefficient of vehicle body were observed during the simulations. In the left bar chart in Fig. 9, there are presented reached values of the lateral shift of individual bogie pivots relatively to relevant bogie frames. The lightened value of $20 \mathrm{~mm}$ represents the proposed lateral clearance of bogie pivots; the value of $40 \mathrm{~mm}$ represents the maximum elastic deformation of proposed bump stops. Under the considered conditions (defined with a relatively high value of unbalanced lateral acceleration), it is evident that the 

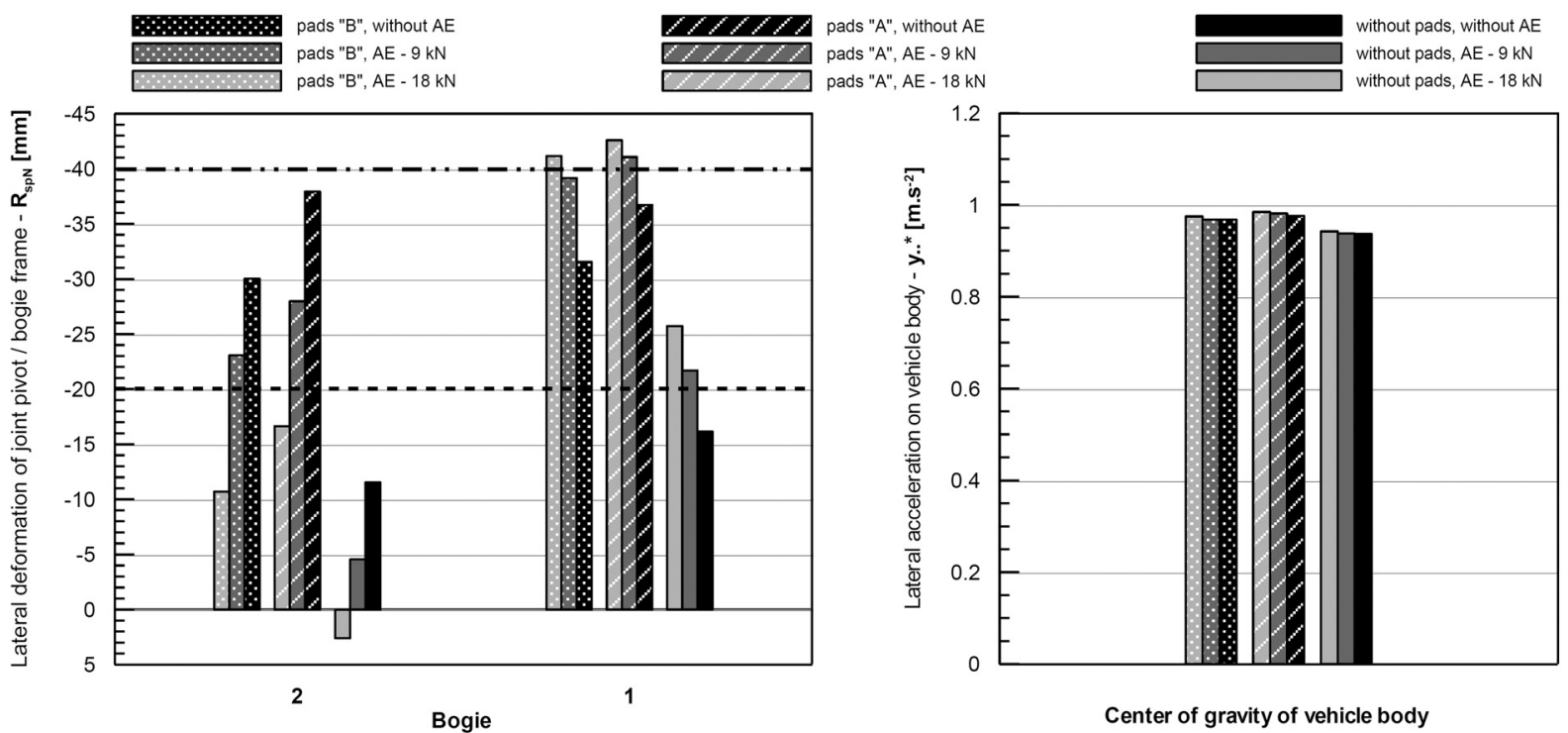

Center of gravity of vehicle body

Fig. 9. Lateral shift of individual bogie pivots of investigated locomotive with the total weight of $138 \mathrm{t}$ relatively to relevant bogie frames (left bar chart) and unbalanced lateral acceleration on the vehicle body (right bar chart) for various versions of locomotive (type of tilting pads, force of active elements) during its run through a right-hand curve with radius of $350 \mathrm{~m}$ at the unbalanced lateral acceleration of $0.8 \mathrm{~m} / \mathrm{s}^{2}$

lateral bogie clearance is not used up only in case of the secondary suspension without the tilting pads. In case of the application of tilting pads, the elastic deformation of lateral bump stops is used. However, the version with the tilting pads "B" shows lower values of lateral shift of the bogie pivots relatively to relevant bogie frames and therefore it can be assumed to be better than the version with the tilting pads " $A$ ".

From the point of view of the roll coefficient of vehicle body, both versions of locomotive with the tilting pads show higher values than the version without pads. The roll coefficient $s_{R}$ can be calculated on basis of the steady value of the lateral acceleration $\ddot{y}^{*}$ measured on the vehicle body (and presented for individual cases in right bar chart in Fig. 9) as

$$
s_{R}=\frac{\ddot{y}^{*}}{a_{n}}-1,
$$

where $a_{n}$ is the unbalanced lateral acceleration. Whereas the considered version of locomotive without the tilting pads shows the roll coefficient of 0.17 , in case of the pads " $A$ " it is 0.22 and 0.21 in case of the pads "B". Therefore, the observed increase of the roll coefficient of vehicle body caused by the tilting pads is acceptable.

\subsection{Influence of active elements for bogie steering on guiding behaviour of locomotive}

In the next stage, an influence of the proposed (optional) system of active elements for bogie steering on guiding behaviour of the locomotive with different arrangement of the secondary suspension (without tilting pads, with pads "A", with pads "B") was investigated. This investigation was performed for two levels of force acting in individual active elements and its results were compared with properties of the locomotive without active elements.

The simulation results demonstrating the influence of active element system on the quasistatic guiding forces acting on individual wheels of the locomotive during its run through the considered curve are presented in Fig. 8. For each investigated version of the locomotive (without tilting pads, with pads "A", with pads "B"), results for a maximum force in the active 
elements of $9 \mathrm{kN}$ and $18 \mathrm{kN}$ are assigned and compared with relevant results obtained with the model of locomotive without the active elements. It is evident that the system of active elements helps to redistribute the guiding forces on individual wheels more evenly — especially a decrease of the force acting on outer wheel of the first wheelset in each bogie is compensated by an increase of the force on outer wheel of the third wheelset. In the considered situation, the reduction of the quasistatic guiding force acting on leading wheels of individual bogies is approximately about $10 \mathrm{kN}$ in case of the locomotive without tilting pads and 12 up to $15 \mathrm{kN}$ in case of the locomotives with tilting pads under the secondary springs. These values were reached for the maximum force in active elements of $18 \mathrm{kN}$; for the half force in the active elements, the reduction of the guiding forces has also approximately half value. In comparison with the relevant version of locomotive without active elements, the total friction power in wheel/rail contact is reduced by approximately $5 \%$ in case of the maximum force in active elements of $9 \mathrm{kN}$ and $10 \%$ in case of the maximum force in active elements of $18 \mathrm{kN}$.

The left bar chart in Fig. 9 demonstrates the influence of active element system on the lateral shift of individual bogie pivots relatively to relevant bogie frames. It is evident that the force action of active elements leads to higher displacements on the front bogie and simultaneously to smaller displacements on the rear bogie. Especially in case of the pads " $A$ " and higher values of the force in active elements, the lateral clearance on the front bogie can be totally used up which is undesirable. The roll coefficient of the vehicle body is not influenced significantly by means of the system of active elements.

\subsection{Assessment of safety against derailment according to GOST standards}

Besides the simulations performed in order to assess the influence of different design solutions of the secondary suspension and the contribution of active elements for bogie steering, further simulations were performed in order to evaluate dynamic properties of the locomotive equipped with the new bogies according to Russian standards (GOST).

Generally, one of the most important quantities, which are assessed in framework of acceptance process of new railway vehicles worldwide, is safety against derailment. Whereas the assessment of safety against derailment during on-track tests according to European standards and UIC leaflets is based on evaluation of the ratio of guiding force and wheel load $Y / Q$ on individual wheels and the maximum value of this ratio should not exceed a defined maximum value (usually 0.8), the Russian standard GOST R 55513-2013 [4] introduces a coefficient $\lambda$ which can be calculated for each wheelset as

$$
\lambda=\frac{C_{1}}{H \cdot 2 s+C_{2} \cdot \mu_{1} \cdot\left(1+0.5 \cdot \mu_{2} \cdot \sin 2 \beta\right)-C_{1} \cdot \mu_{2} \cdot \cos ^{2} \beta} \cdot \frac{1}{\operatorname{cotg} \beta+\mu_{2}},
$$

where quantities $C_{1}$ and $C_{2}$ are defined as:

$$
\begin{aligned}
& C_{1}=2 \cdot B_{0} \cdot\left(b-a_{1}\right)-F_{f w z 2} \cdot\left(2 s+a_{2}\right)+F_{f w z 1} \cdot a_{1}+H \cdot r+G_{u s m} \cdot\left(b-a_{1}\right), \\
& C_{2}=2 \cdot B_{0} \cdot\left(b-a_{2}\right)-F_{f w z 1} \cdot\left(2 s+a_{1}\right)+F_{f w z 2} \cdot a_{2}+H \cdot r+G_{u s m} \cdot\left(b-a_{2}\right)
\end{aligned}
$$

and individual symbols have following meaning: $B_{0}$ is a static load of individual axle boxes, $F_{f w z i}$ are dynamic forces in primary suspension, $G_{u s m}$ is a weight of unsuspended masses per wheel, $H$ is a frame force, $2 s$ is a distance between rolling circles, $r$ is a nominal wheel radius, $b$ is a half of lateral distance between centres of axle boxes, dimensions $a_{i}$ represent a distance between relevant centre of axle box and plane of rolling circle, $\beta$ is a flange angle and values of $\mu_{i}$ represent friction coefficient in wheel/rail contact. The quantities with subscript 1 


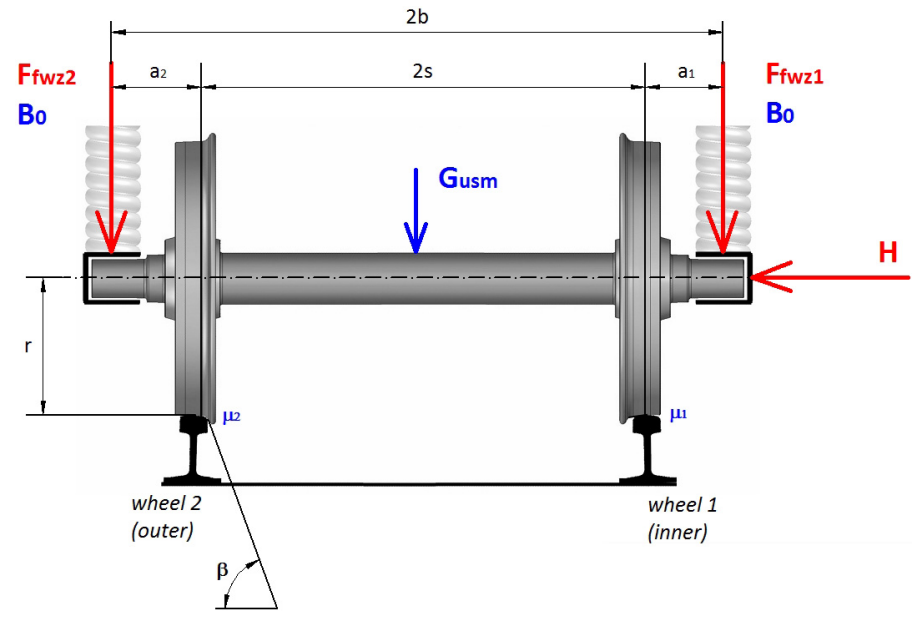

Fig. 10. Scheme of forces acting on a wheelset and used for assessment of safety against derailment by means of coefficient $\lambda$ according to GOST R 55513-2013 [4]

are related to the inner wheel (wheel 1); subscript 2 refers to the outer wheel (wheel 2). The considered parameters are depicted in Fig. 10.

On basis of the simulation results, behaviour of the coefficient $\lambda$ can be calculated for all wheelsets of the investigated vehicle. In Fig. 11, there are presented relevant results for the first wheelset of the locomotive with different arrangements of the secondary suspension (without tilting pads, with pads "A", with pads "B") and without the system of active elements for bogie steering. These results were obtained at simulation of run of the vehicle on a track with real (measured) track irregularities. It is evident that the lowest values shows the locomotive without tilting pads (mean value in the curve: 2.41 , standard deviation: 0.09 , minimum value: 2.18 ); in case of the locomotive with pads "A"/"B", these values are following - mean: $2.57 / 2.53$, standard deviation: $0.10 / 0.10$, minimum: $2.32 / 2.29$.

The limit value of $\lambda$ according to [4] is defined as 1.4 and the measured values should not be lower. Therefore, the results of all investigated variants meet this requirement.

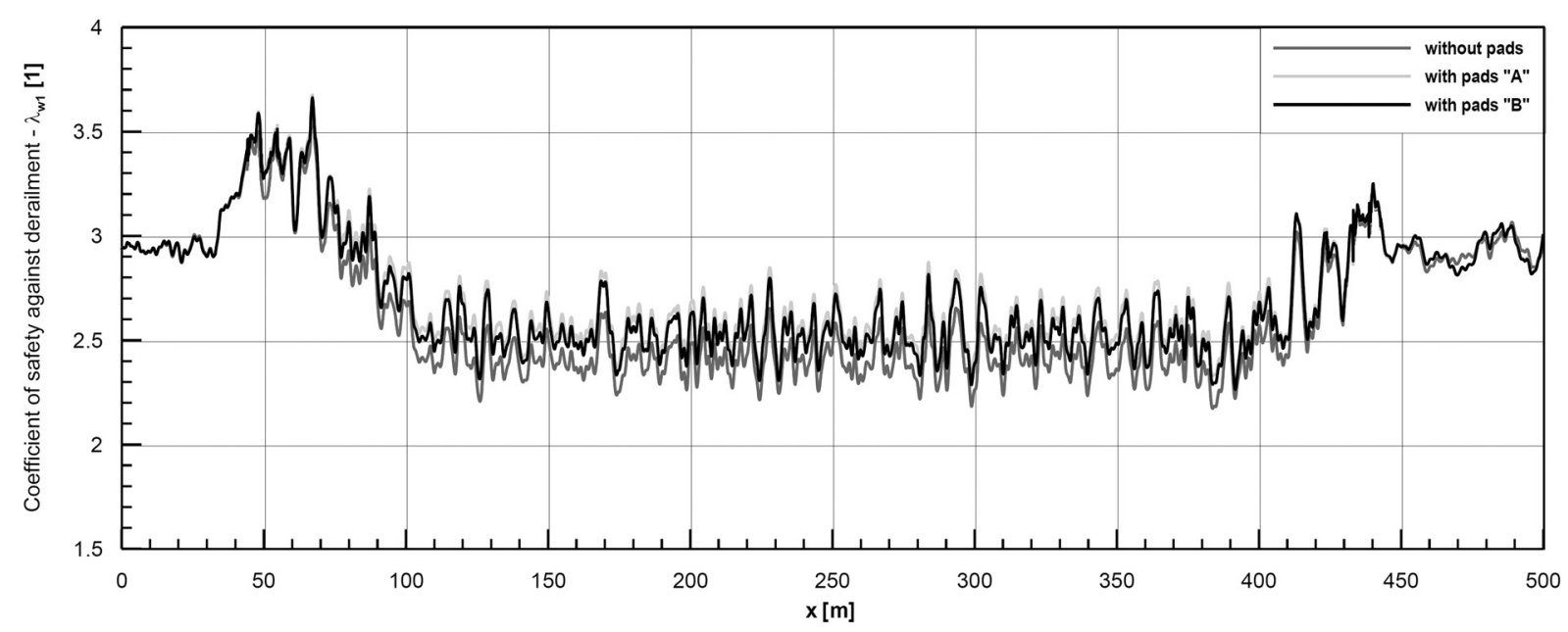

Fig. 11. Behaviour of coefficient $\lambda$ for assessment of safety against derailment according to GOST R 55513-2013 [4] for the first wheelset of the investigated locomotive with the total weight of $138 \mathrm{t}$ in various versions (defined with the type of tilting pads) during its run through a right-hand curve with radius of $350 \mathrm{~m}$ at the unbalanced lateral acceleration of $0.8 \mathrm{~m} / \mathrm{s}^{2}$ 


\section{Conclusion}

This paper deals with an assessment of influence of different types of tilting rubber-metal pads under secondary flexi-coil springs of a locomotive equipped with new three-axle bogies on guiding behaviour of the vehicle in small-radius curves, including an example of assessment of safety against derailment according to GOST standards. An effect of a system of active elements for bogie steering on dynamic properties of the locomotive and its usability is also discussed. On bases of the presented results, following conclusions can be stated:

- The considered tilting pads under the secondary flexi-coil springs reduce the bogie yaw resistance significantly and therefore their application leads to a decrease of quasistatic guiding force acting on outer wheel of the first wheelset in small-radius curves. In the investigated cases, this guiding force reduction caused by application of the tilting pads has a value of approximately $7 \%$.

- The tilting pads under the secondary springs also reduce the lateral stiffness of the secondary suspension and therefore their application leads to higher lateral displacements between the vehicle body and the bogie frames under given conditions (unbalanced lateral acceleration, mass of vehicle body). Therefore, the overall lateral stiffness characteristic of the joint vehicle body/bogie frame (consisting of the flexi-coil springs with pads and lateral bump stops) has to be optimized for particular application (i.e. for mass and dimensional parameters of the vehicle).

- The proposed system of active elements for bogie steering allows a very significant reduction of the guiding forces acting on outer wheels of leading wheelsets in both bogies. In case of the first wheelset, the guiding force can be reduced by approximately 15 up to $20 \%$. A reduction of the total friction power in wheel/rail contact up to $10 \%$ can be also reached. However, the proposed system of active bogie steering disposing with high forces in active elements can be simply applied only on a locomotive without tilting pads under the secondary spring. In case of a locomotive equipped with the considered tilting pads (especially of the type "A"), a system of active lateral suspension would have to be supplemented to minimize the lateral deformation of secondary suspension, or only limited values of forces in active elements (with respect to the vehicle parameters) should be applied.

\section{Acknowledgements}

The work has been supported by the grant project No. TH01010455 "Research and Development of a Three-axle Bogie for the Track Gauge 1520 mm" of the Technology Agency of the Czech Republic.

\section{References}

[1] Baur, K. G., Drehgestelle — Bogies, EK-Verlag, Freiburg, 2006.

[2] Bruni, S., Vinolas, J., Berg, M., Polach, O., Stichel, S., Modelling of suspension components in a rail vehicle dynamics context, Vehicle System Dynamics 49 (7) (2011) 1021-1072. https://doi.org/10.1080/00423114.2011.586430

[3] GOST 11018-2011. Traction railway stock wheelsets of $1520 \mathrm{~mm}$ gauge railways. General specifications. (in Russian)

[4] GOST R 55513-2013. Locomotives - Requirements for strength and dynamic properties. (in Russian) 
[5] Haupt, L., Kopal, J., Michálek, T., Štěpánek, J., Benický, M., Staněk, P., Čejka, T., Liberová, S., Development of a three-axle bogie for diesel-electric locomotive, Proceedings of the 23rd conference with international participation Current Problems in Rail Vehicles 2017, Česká Třebová, University of Pardubice, Faculty of Transport Engineering, 2017, pp. 141-150.

[6] Haupt, L., Michálek, T., A three-axle locomotive bogie. Utility model No. 31311. Praha, Industrial Property Office, registration date: 18/12/2017.

[7] Jiang, Y., Lateral stiffness simplified calculation for flexicoil spring with rubber pad on one end of railway locomotive and rolling stock, Applied Mechanics and Materials 525 (2014) 214-217. https://doi.org/10.4028/www.scientific.net/AMM.525.214

[8] Michálek, T., Vágner, J., Kohout, M., Zelenka, J., Analytical calculation and experimental verification of lateral stiffness of a flexi-coil spring with tilting rubber-metal pad, Scientific Papers of the University of Pardubice, Series B - The Jan Perner Transport Faculty 19 (2014) 167-179.

[9] Michálek, T., Zelenka, J., Modelling of flexi-coil springs with rubber-metal pads in a locomotive running gear, Applied and Computational Mechanics 9 (2015) 21-30.

[10] Michálek, T., Zelenka, J., Reduction of lateral forces between the railway vehicle and the track in small-radius curves by means of active elements, Applied and Computational Mechanics 5 (2011) 187-196.

[11] Spiryagin, M., Wolfs, P., Cole, C., Spiryagin, V., Sun, Y. Q., McSweeney, T., Design and simulation of heavy haul locomotives and trains, CRC Press, Boca Raton, 2017.

[12] Zelenka, J., Running and guiding behaviour of two-axled diesel-electric locomotives CZ LOKO, New Railway Technique 6 (2009) 15-23. (in Czech) 